\title{
Comparison of Pregnane X Receptor Antagonists for Enhancing the Antitumor Effect of Cisplatin
}

\author{
MEGUMI YASUDA $^{1}$, SHUICHI KISHIMOTO ${ }^{2}$, MANABU AMANO ${ }^{1}$ and SHOJI FUKUSHIMA ${ }^{2}$ \\ ${ }^{1}$ Department of Pharmacy, School of Pharmacy, Hyogo University of Health Sciences, Kobe, Japan; \\ ${ }^{2}$ Department of Pharmaceutics, Faculty of Pharmaceutical Sciences, Kobe Gakuin University, Kobe, Japan
}

\begin{abstract}
Background: Cisplatin is a platinum compound capable of inducing apoptosis of cancer cells. However, cancer cells can become cisplatin-resistant. A recent study showed that a pregnane $X$ receptor (PXR) antagonist, leflunomide, can enhance the antitumor activity of cisplatin and overcome such resistance. This study determined whether PXR antagonists ketoconazole and phenethyl isothiocyanate (PEITC) enhance the antitumor activity of platinum compounds and by which mechanism(s) of action. Materials and Methods: Caspase-3 activity, intracellular platinum level, and expression of ATP-binding cassette subfamily $C$ member 2 (ABCC2; previously named multidrug resistance-associated protein 2) were assessed in HepG2 human hepatocellular carcinoma cells exposed to carboplatin or cisplatin with and without PXR antagonist. Results: In combination with platinum compounds, PEITC increased the intracellular platinum level, while ketoconazole induced higher caspase-3 activity. Additionally, PEITC suppressed ABCC2 protein expression. Conclusion: These results suggested that ketoconazole and PEITC enhance the antitumor activity of platinum compounds by different and complex mechanisms.
\end{abstract}

Pregnane $\mathrm{X}$ receptor (PXR) is a nuclear receptor discovered in 1998 by several groups. PXR regulates the detoxification mechanism of many xenobiotics through the transcriptional regulation of many metabolic enzymes and transporters (1). Target genes regulated by PXR include cytochrome P450 (CYP) 3A4 (2), CYP2B6 (3), UDP-glucuronosyltransferases family (4) for metabolic enzymes, ATP-binding cassette subfamily $\mathrm{C}$ member (ABCC) $-1,-2$, and $-3(5,6)$, and organic anion transporting polypeptide 2 (7) for transporters.

Correspondence to: Shuichi Kishimoto, Department of Pharmaceutics, Faculty of Pharmaceutical Sciences, Kobe Gakuin University, Minatojima 1-1-3, Chuo-ku, Kobe 650-8586, Japan. Tel: +81 789744753, Fax: +81 789744753, e-mail: skisimot@pharm. kobegakuin.ac.jp

Key Words: Cisplatin, pregnane $\mathrm{X}$ receptor, transporter, apoptosis.
Although PXR is mainly expressed in the liver and intestinal tract, its expression in tumors is still unknown. The expression of various genes involved in the metabolism and excretion of anticancer drugs, such as all-trans retinoic acid (8), etoposide (9) and paclitaxel (10) is also regulated by PXR, thus the potential role of PXR in anticancer drug resistance has attracted attention.

Cisplatin is a platinum compound capable of inducing cell apoptosis when used in the treatment of many types of solid cancer. However, acquisition of cisplatin resistance in cancer cells has become an important clinical issue. To this end, various mechanisms involved in such resistance have been identified, such as reduced intracellular cisplatin uptake, augmented extracellular cisplatin excretion, and increased activity of detoxification and nucleic acid repair systems. Many cisplatin-resistant cell lines also showed a decrease in intracellular platinum level, which was associated with a decrease in its intracellular uptake or increase in its extracellular excretion $(11,12)$, besides an increase in ABCC2 expression level $(13,14)$. As we previously mentioned, ABCC2 expression is regulated by PXR $(15,16)$, and cisplatin-resistant cells may present increased PXR expression/activity.

Bitter melon extract, a PXR antagonist, was shown to enhance the cytotoxicity of doxorubicin in a concentrationdependent manner by suppressing the activity of PXR and the expression of another ATP binding cassette subfamily B member 5, P-glycoprotein (ABCB5) which is a major efflux transporter of doxorubicin in cancer cells (17). We also reported that the PXR antagonist, leflunomide, enhanced the cytotoxicity of cisplatin in the cisplatin-resistant HepG2/R cell line (18). Thus, PXR antagonists have the potential to enhance the effects of anticancer agents by suppressing the expression of efflux transporters.

Ketoconazole is an imidazole that has been used since the 1980 s to treat various fungal infections (19). Additionally, ketoconazole was shown to be effective in treating prostate cancer (20), but its molecular mechanism is unknown. It has also been reported that ketoconazole has an inhibitory action on drug-metabolizing enzymes such as CYP450s by blocking the activation of nuclear receptors $(21,22)$. Isothiocyanate is 
formed by the decomposition of glucosinolate, which is the main component of cruciferous vegetables (watercress, cabbage, Brussel sprouts, cauliflower, etc.). Specifically, phenethyl isothiocyanate (PEITC), inhibited CYP3A4 expression via PXR, and midazolam clearance mediated by CYP3A4 (23). In this study, we hypothesized that ketoconazole and PEITC, both PXR antagonists, might also further enhance the cytotoxicity of cisplatin. Therefore, we compared the effects of ketoconazole and PEITC on the cytotoxicity and intracellular accumulation of platinum compounds, and examined the association between PXR antagonism that suppressed $\mathrm{ABCC} 2$ expression and the enhanced effects of platinum compounds.

\section{Materials and Methods}

Cell culture. Human hepatocellular carcinoma HepG2 cells were purchased from Dainippon Pharmaceutical (Osaka, Japan). Cells were maintained in minimum essential medium (Nacalai Tesque, Kyoto, Japan) containing $10 \%$ fetal bovine serum at $37^{\circ} \mathrm{C}$ in humidified air with $5 \% \mathrm{CO}_{2}$.

Caspase-3 assay. Caspase-3 enzyme activity was measured by proteolytic cleavage of the fluorogenic substrate $\mathrm{N}$-acetyl-AspGlu-Val-Asp-7-amido-4-trifluoromethylcoumarin (Ac-DEVDAFC) using Caspase-3 Fluorometric Assay Kit (BioVison, CA, USA). HepG2 cells were plated at a density of $2.0 \times 10^{5}$ cells per well into 6-well plates on day 0 . On day 1, $30 \mu \mathrm{M} \mathrm{KTZ} \mathrm{(Nacalai}$ Tesque) or PEITC (Nacalai Tesque) were added to each well. Finally, on day $3,10 \mu \mathrm{M}$ cisplatin or $180 \mu \mathrm{M}$ carboplatin were added to except for the wells of control and PXR antagonist alone. After $72 \mathrm{~h}$ from plating $(24 \mathrm{~h}$ in the presence of platinum compound), the cells were harvested and washed with cold phosphate-buffered saline (PBS). The pellets were lysed using 100 $\mu \mathrm{l}$ of cell lysis buffer, and then placed on ice. A small portion $(5 \%)$ of the cell lysate was used to determine the protein content using a BCA Protein Assay Kit (Pierce, IL, USA). The cell lysates were adjusted to a protein concentration of $0.25 \mu \mathrm{g} / \mu \mathrm{l}$ with cell lysis buffer. The reaction mixtures were prepared in a 96-well plate and contained the following components: $50 \mu \mathrm{l}$ of the sample, $50 \mu \mathrm{l}$ of $2 \mathrm{X}$ reaction buffer containing $10 \mathrm{mM}$ dithiothreitol, and $5 \mu \mathrm{l}$ of $2 \mathrm{mM}$ Ac-DEVD-AFC solution. The reaction mixtures were incubated at $37^{\circ} \mathrm{C}$ for $1 \mathrm{~h}$ and at room temperature for another hour. The fluorescence was then measured on a fluorescence reader (Versa Fluor fluorometer; Bio-Rad Laboratories, Hercules, CA, USA), and expressed as a relative value with respect to the control group.

Intracellular platinum accumulation. HepG2 cells were seeded into $75-\mathrm{cm}^{2}$ tissue culture flasks at a cell density of $1.0 \times 10^{6}$ per flask on day 0 . On day $1,30 \mu \mathrm{M} \mathrm{KTZ} \mathrm{or} \mathrm{PEITC} \mathrm{was} \mathrm{added,} \mathrm{and} \mathrm{finally} \mathrm{on}$ day $225 \mu \mathrm{M}$ cisplatin or $180 \mu \mathrm{M}$ carboplatin were then added to them. After 24-h exposure to platinum compound, the cells were harvested and washed three times with cold PBS. A small proportion (5\%) of the cells from each experimental group was used to determine the protein content using a BCA Protein Assay Kit (Pierce). The remaining cells were suspended in $1.5 \mathrm{ml}$ of a mixture of perchloric acid and $30 \%$ hydrogen peroxide at the ratio of $1: 2$ and digested for $12 \mathrm{~h}$ at $65^{\circ} \mathrm{C}$. The amount of platinum in the samples was determined using an SPS 3100 Inductively Coupled Plasma Optical Emission Spectrometer (ICP-OES; Hitachi HighTech Science Corporation, Tokyo, Japan). The results were expressed as nanograms of platinum per milligram of cell protein.

RNA extraction and real-time polymerase chain reaction ( $R T-P C R)$. HepG2 cells were seeded into $75-\mathrm{cm}^{2}$ tissue culture flasks at a cell density of $1.0 \times 10^{6}$ per flask on day 0 . On day $1,30 \mu \mathrm{M}$ ketoconazole or PEITC were added, and on day 2, $10 \mu \mathrm{M}$ cisplatin was added to the corresponding flasks. After 48-h exposure to ketoconazole or PEITC, the cells were harvested and washed three times with cold PBS. Total RNA was extracted using FastGene Premium Kit (NIPPON Genetics, Tokyo, Japan) in accordance with the manufacturer's protocol. The quality and concentration of RNA in each sample was confirmed by spectrophotometry using NanoDrop Lite (Thermo Fisher Scientific, Yokohama, Japan). Copies of cDNA were produced from total RNA by reverse transcription using a PrimeScript II First Strand cDNA Synthesis Kit (Takara Bio, Shiga, Japan). Amplification of DNA sequences was performed using Rotor-Gene Q (Qiagen, Tokyo, Japan) with a KAPA SYBR Fast qPCR Kit (NIPPON Genetics), and normalized to that of glyceraldehyde 3-phosphate dehydrogenase $(G A P D H)$ to obtain relative expression levels. The primers for RT-PCR, synthesized by BEX (Tokyo, Japan), were designed as follows: ABCC2: forward 5'-ACGGGCACATCACCATCAAG-3', reverse 5'-CTCCAGGCAGCATTTCCAAG-3'; GAPDH: forward 5'-AAC GGGAAGCTTGTCATCAATGGAAA-3'; reverse 5'-GCATCA GCAGAGGGGGCAGAG-3'. The PCR conditions were set as follows: $95^{\circ} \mathrm{C}$ for $5 \mathrm{~s}$ and $60^{\circ} \mathrm{C}$ for $10 \mathrm{~s}, 40$ cycles. The cycle threshold (CT) indicated the fractional cycle number at which the PCR product was first detected above a fixed threshold. Relative mRNA levels were determined using the $2^{-\Delta \Delta \mathrm{CT}}$ method.

Western blotting. HepG2 cells were seeded into $75-\mathrm{cm}^{2}$ tissue culture flasks at a cell density of $1.0 \times 10^{6}$ per flask on day 0 and $30 \mu \mathrm{M}$ ketoconazole or PEITC were added on day 1 . After $24 \mathrm{~h}$ exposure to ketoconazole or PEITC, the cells were harvested and washed three times with cold PBS. Cell membrane protein fractions were extracted using a plasma membrane protein extraction kit (MBL, Tokyo, Japan) according to the manufacturer's protocols. Proteins $(20 \mu \mathrm{g})$ were subjected to sodium dodecyl sulfate-polyacrylamide gel electrophoresis and transferred to polyvinylidene difluoride membrane. The membrane was blocked with Bullet Blocking One for Western Blotting (Nacalai Tesque) for $1 \mathrm{~h}$ at room temperature and then incubated overnight at $4^{\circ} \mathrm{C}$ with monoclonal antibody against $\mathrm{ABCC} 2$ (24893-1-AP, Proteintech, IL, USA), or GAPDH (10494-1-AP, Proteintech). The membrane was rinsed with PBS-T (FUJIFILM Wako Pure Chemical, Osaka, Japan) and then incubated for $1 \mathrm{~h}$ with horseradish peroxidase-conjugated anti-rabbit IgG (\#7470; Cell Signaling Technology, Danvers, MA, USA). The membrane was washed, and the protein bands were visualized with ECL Prime Western Blotting Detection Reagent (GE Healthcare Japan, Tokyo, Japan) according to the manufacturer's protocols.

Statistical analysis. Results are expressed as means \pm standard deviation (SD). Statistical analysis was performed with Microsoft Excel (Microsoft, Redmond, WA, USA) using Dunnett's test for multiple comparisons with a control group. A value of $p<0.05$ was considered statistically significant. 
A

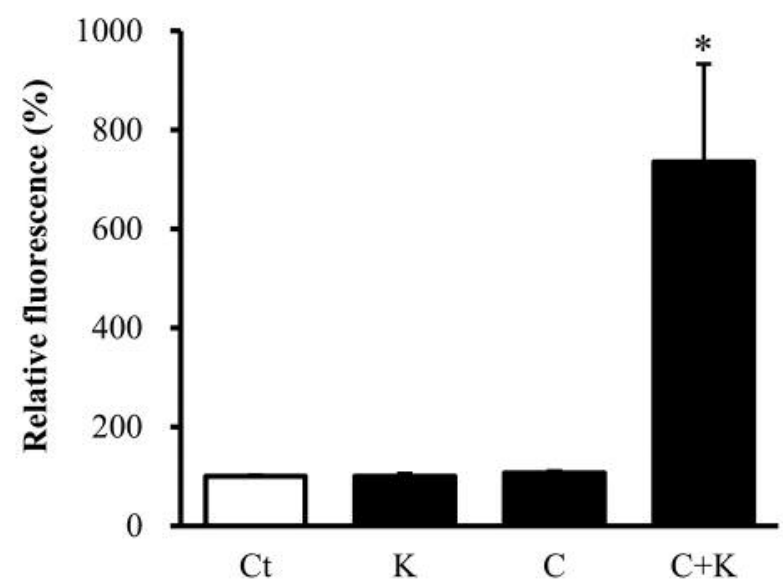

C

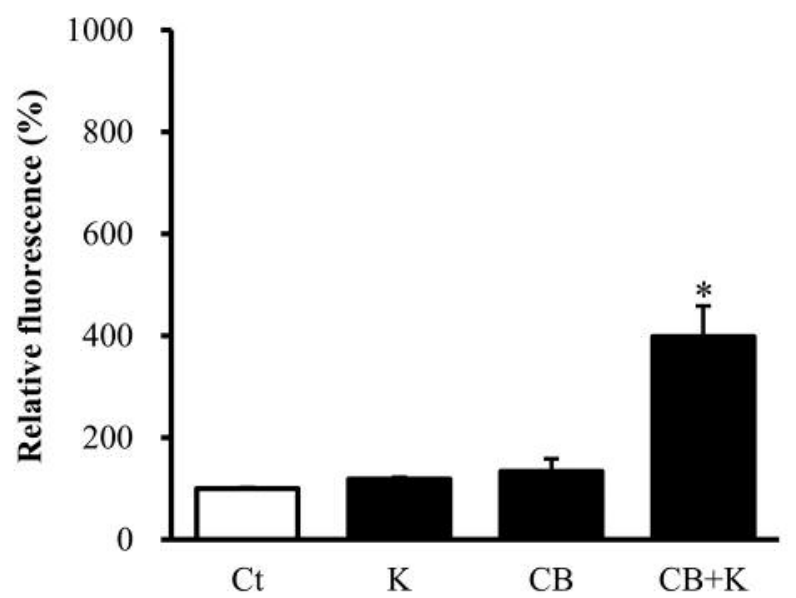

B

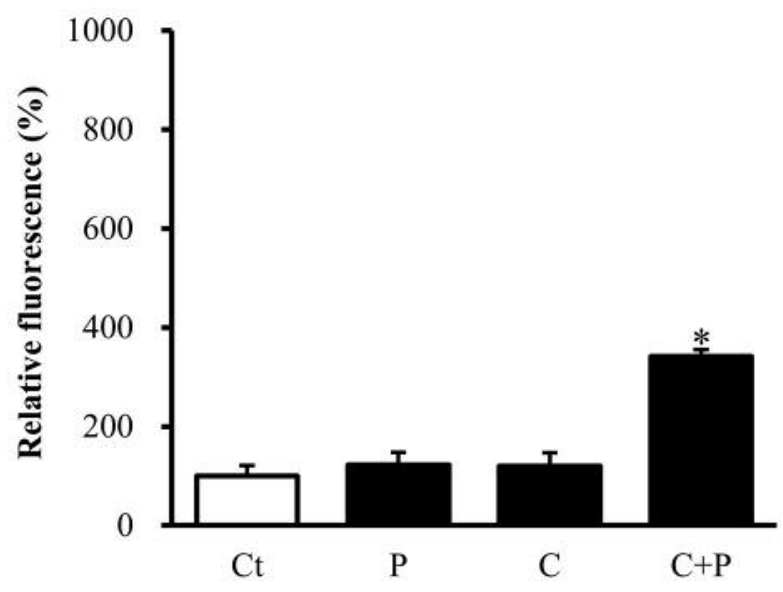

D

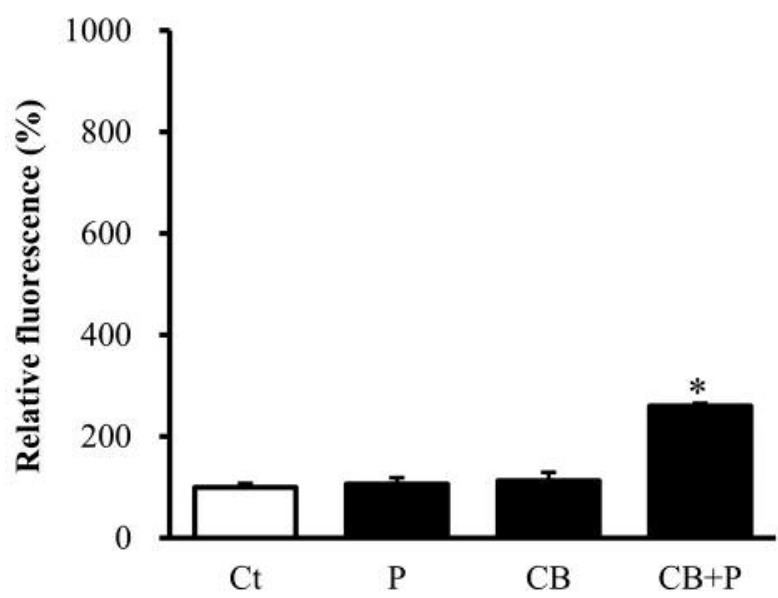

Figure 1. Effects of ketoconazole $(K)$ and phenethyl isothiocyanate (PEITC) $(P)$ on caspase-3 activation induced by cisplatin $(C)$ or carboplatin $(C B)$ in HepG2 cells. Cells were exposed to $30 \mu M$ of ketoconazole or PEITC for 48 h with/without $10 \mu M$ of cisplatin (A and B, respectively) or $180 \mu \mathrm{M}$ of carboplatin (C and D, respectively) for $24 \mathrm{~h}$. Caspase-3 activity in HepG2 cells was assessed in each group by fluorescence and expressed as the fold increase with respect to the untreated control group $(C t)$. Data are presented as the mean $\pm S D$ for three independent experiments. *Significantly different at $p<0.05 \mathrm{vs}$. control.

\section{Results}

Effect on caspase-3 activity. In our previous study (18), cisplatin significantly increased caspase-3 activity at $25 \mu \mathrm{M}$ in HepG2 cells, as compared with $10 \mu \mathrm{M}$. Similarly, carboplatin increased caspase-3 activity at $360 \mu \mathrm{M}$ in HepG2 cells but had no effect at $180 \mu \mathrm{M}$. Therefore, the effect on caspase- 3 activity in combination with PXR antagonists was examined in this study using $10 \mu \mathrm{M}$ cisplatin and $180 \mu \mathrm{M}$ carboplatin. On the other hand, ketoconazole and PEITC alone did not affect caspase-3 activity at $30 \mu \mathrm{M}$ in HepG2 cells. However, it significantly increased it when used in combination with cisplatin or carboplatin (Figure 1). For both cisplatin and carboplatin, the combination with PEITC resulted in a greater increase of caspase- 3 activity. However, the effect of PXR antagonists on caspase- 3 activity was lower in presence of carboplatin compared to cisplatin.

Effect on platinum accumulation. When HepG2 cells were exposed to $25 \mu \mathrm{M}$ cisplatin or $360 \mu \mathrm{M}$ carboplatin alone, the intracellular platinum levels were $110 \pm 4.7$ and $222 \pm 55.8$ $\mathrm{ng} / \mathrm{mg}$ protein, respectively (Figure 2). Therefore, the intracellular platinum level per molar dose exposure were much lower when in the presence of carboplatin compared to 
A

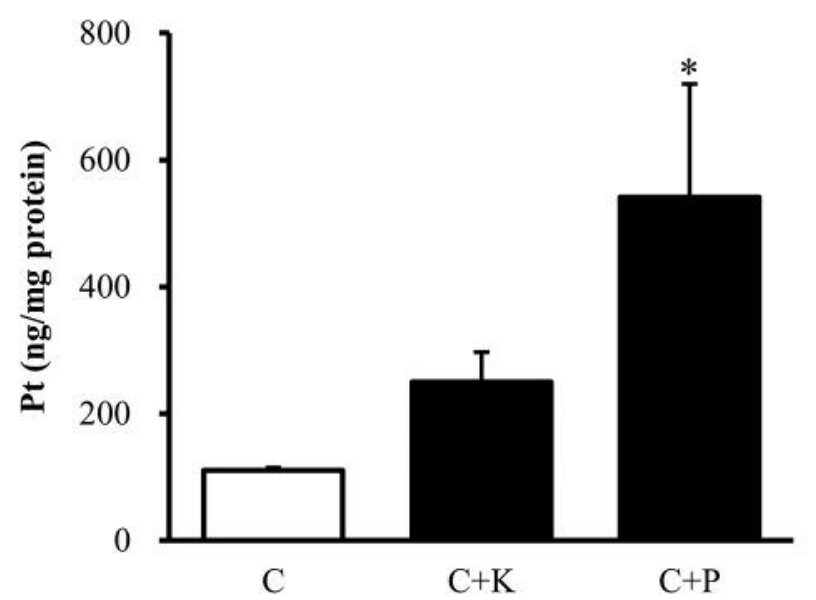

B

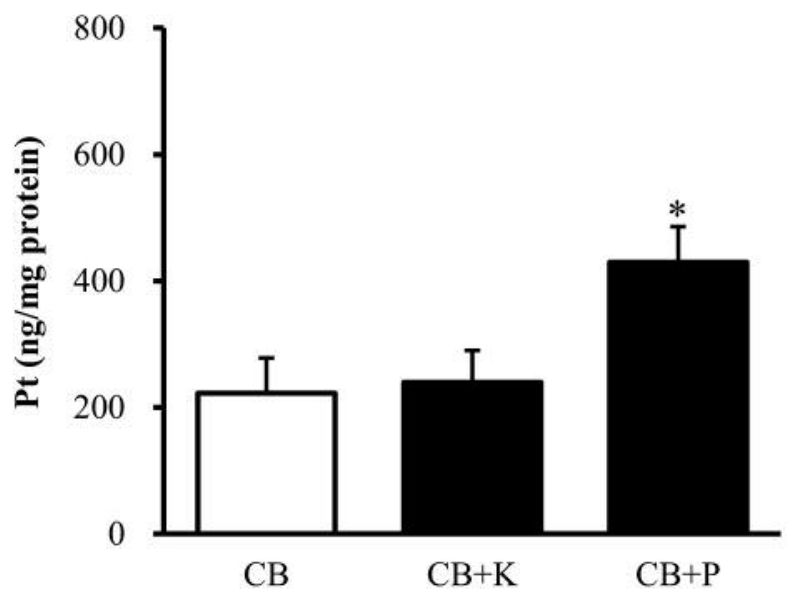

Figure 2. Effects of ketoconazole $(K)$ and phenethyl isothiocyanate (PEITC) $(P)$ on the intracellular platinum accumulation in HepG2 cells additionally exposed to cisplatin $(C)(A)$ or carboplatin $(C B)(B)$ for 24 h. The control group was exposed to cisplatin or carboplatin only. Data are presented as mean $\pm S D$ of three independent experiments. *Significantly different at $p<0.05 \mathrm{vs}$. control.

cisplatin. When cisplatin was used in combination with PXR antagonist, the intracellular platinum level increased 2.3 and 4.9 times with ketoconazole and PEITC, respectively. Moreover, administration of carboplatin in combination with ketoconazole did not change the intracellular platinum level but when combined with PEITC increased it by 1.9 -fold. Thus, the combined effect of PXR antagonists on the intracellular platinum level was lower with carboplatin compared to cisplatin.

Effect on ABCC2 mRNA expression. The effects of ketoconazole and PEITC on the mRNA expression level of $A B C C 2$, a transporter regulated by PXR, which extracellularly expels platinum compounds, was examined (Figure 3). Ketoconazole did not affect the expression of $A B C C 2$ mRNA, neither alone nor in combination with 10 $\mu \mathrm{M}$ cisplatin. However, PEITC significantly reduced the $A B C C 2$ mRNA expression level to $18 \%$ when used in combination with $10 \mu \mathrm{M}$ cisplatin. On the other hand, 10 $\mu \mathrm{M}$ cisplatin alone showed no effect, but $25 \mu \mathrm{M}$ cisplatin alone did significantly reduce its expression level (data not shown).

Effect on ABCC2 protein expression. The effect of PXR antagonist on expression of $\mathrm{ABCC} 2$ protein in the cell membrane fraction of HepG2 cells was analyzed (Figure 4). After exposure of cells to $30 \mu \mathrm{M}$ PXR antagonist for $24 \mathrm{~h}$, no effect was observed in the presence of ketoconazole, but the ABCC2 expression level was significantly reduced to $18 \%$ with PEITC exposure.

\section{Discussion}

One of the factors involved in acquiring resistance to cisplatin is a reduction in intracellular platinum accumulation. Decreased intracellular platinum level result from reduced intracellular uptake, enhanced extracellular export, or both (11, 12). Various transporters play an important role in these pathways. Among them, ABCC2 has been associated with cisplatin resistance $(13,14)$. Nuclear receptors are known to be involved in the regulation of transporter expression, such as the expression of ABCC2, which is regulated by PXR (15, 16). Previous studies have shown that rifampicin, a PXR agonist, suppressed the cytotoxicity of cisplatin, and that treatment with the PXR antagonist leflunomide overcame cisplatin resistance (18). Other reports also suggested that coadministration of PXR antagonist might improve the efficacy of antitumor drugs by inhibiting the acquisition of multi-drug resistance (17).

In this study, we investigated the effects of two structurally different PXR antagonists, ketoconazole, an azole antifungal agent, and PEITC, an isothiocyanate derivative, to determine whether the combination of platinum compounds with PXR antagonists enhances antitumor activity. Carboplatin and cisplatin were also evaluated as platinum compounds. Ketoconazole and PEITC both significantly increased caspase3 activity in combination with cisplatin, although ketoconazole showed stronger enhancement. The intracellular platinum level increased when each PXR antagonist and cisplatin were used in combination, but the use of PEITC resulted in a larger increase. Even when used in combination with carboplatin, 

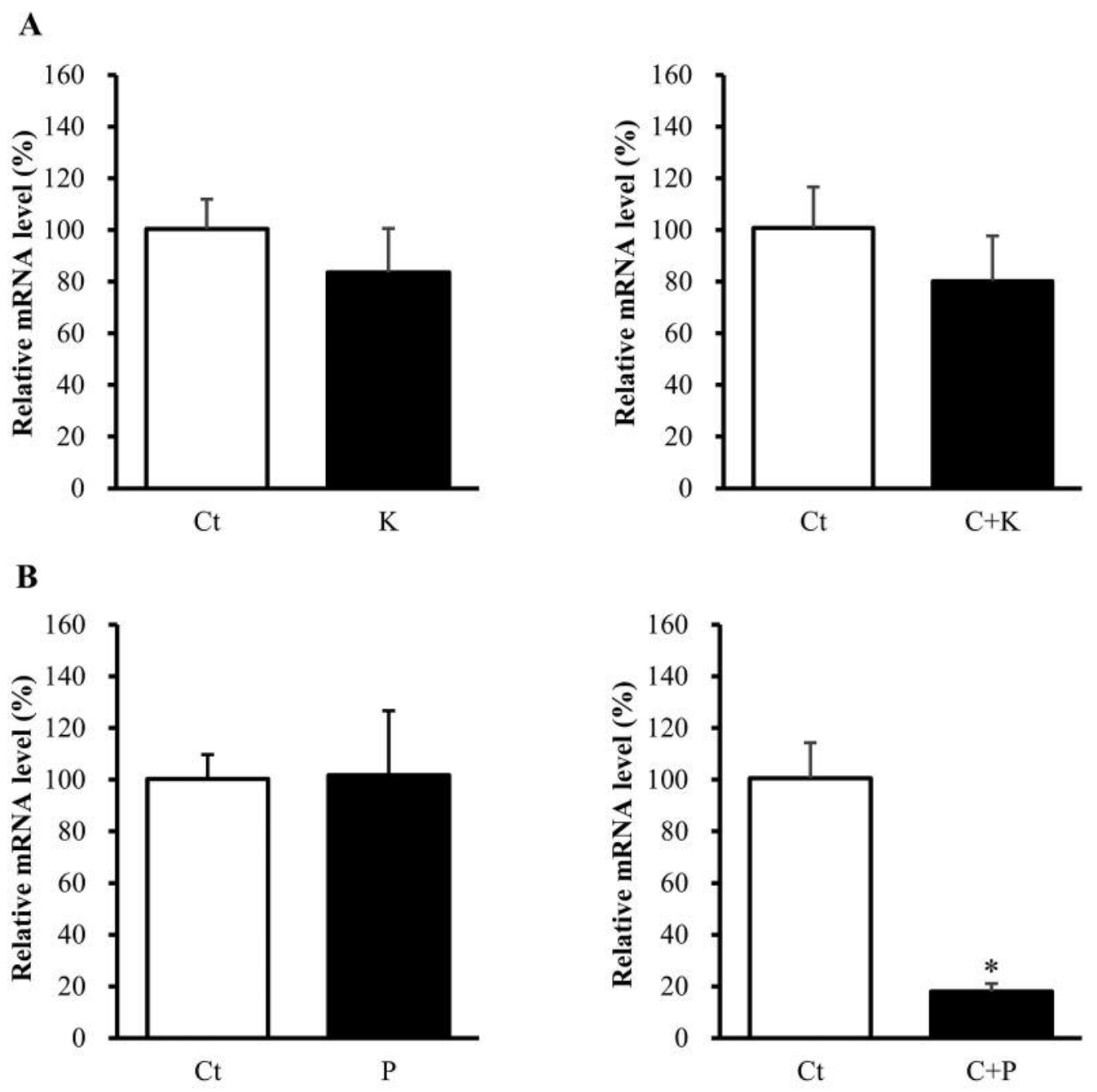

Figure 3. Relative ATP-binding cassette subfamily C member 2 (ABCC2) mRNA level in HepG2 cells exposed to ketoconazole (K) and phenethyl isothiocyanate $(P E I T C)(P)$ alone and in combination with cisplatin $(C)$ ( $A$ and B, respectively). ABCC2 mRNA level was normalized to that of glyceraldehyde 3-phosphate dehydrogenase (GAPDH). The relative mRNA level was expressed as the fold increase with respect to that of the control group $(C t)$. Data are presented as means $\pm S D$ of three independent experiments. *Significantly different at $p<0.05$ vs. control.

ketoconazole exerted a pronounced increase in caspase-3 activity. However, concomitant administration of PEITC increased the intracellular platinum level even further. Thus, we observed that ketoconazole and PEITC enhanced the intracellular platinum level by the combination with cisplatin or carboplatin, but the increased effect was not correlated with the increase in intracellular platinum level. In general, the antitumor activity of platinum compounds is dependent on the level of platinum accumulated in cells. Therefore, both these PXR antagonists may operate through different mechanisms of action. In this study, we assumed that the mechanism of PXR antagonist was suppression of the expression of ABCC2, which could act as an efflux transporter of platinum compounds. According to our results, PEITC suppressed
ABCC2 membrane protein expression, whereas ketoconazole did not, suggesting that the enhancement of the cisplatin activity by PEITC was largely attributed to the increase in intracellular platinum accumulation due to the suppression of ABCC2 expression. However, ABCC2 mRNA levels were not changed by PEITC alone, but a marked decrease was observed when it was combined with cisplatin. On the other hand, ketoconazole did not affect $A B C C 2$ mRNA level even in combination with cisplatin, in contrast with PEITC. Tang et al. reported that $A B C B 1$ and $A B C C 1$, whose expression was regulated by $\mathrm{PXR}$, were down-regulated at mRNA and protein levels in SGC7901/DDP human cisplatin-resistant gastric cancer cells treated with PEITC (24). Moreover, PEITC was reported to be a substrate of $\mathrm{ABCC} 2$, and to interact with 
A

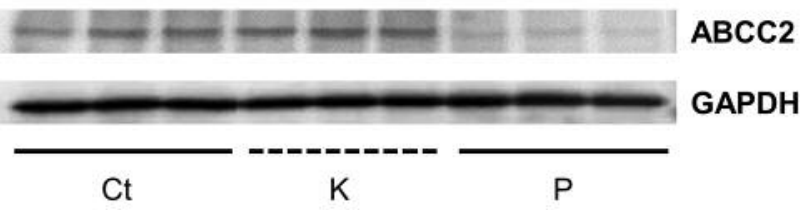

B

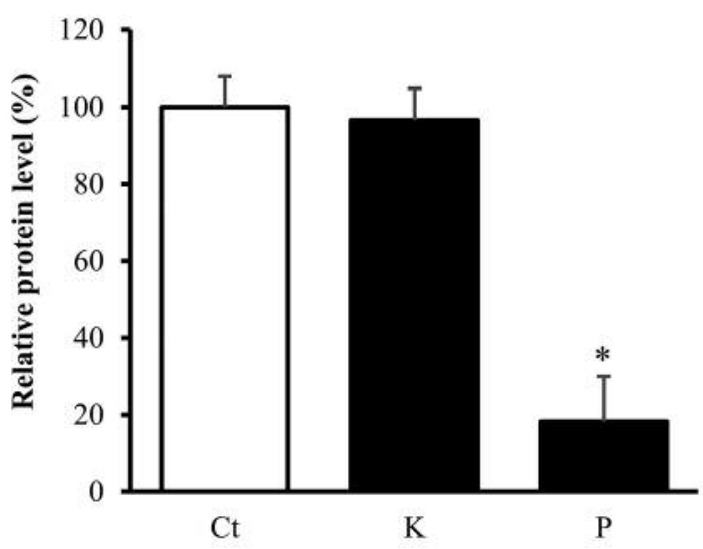

Figure 4. Membrane protein expression level of ATP-binding cassette subfamily $C$ member 2 ( $A B C C 2)$ in HepG2 cells exposed to ketoconazole $(K)$ and phenethyl isothiocyanate $(P E I T C)(P)$ alone and in combination with cisplatin $(C)$ or carboplatin $(C B)$. A: Immunoblotting for $A B C C 2$ and glyceraldehyde 3-phosphate dehydrogenase (GAPDH) in HepG2 cells. B: Protein levels of ABCC2 were normalized to that of GAPDH. Relative protein level of $A B C C 2$ is expressed as fold increase with respect to that of the untreated control group. Data are presented as mean $\pm S D$ of three independent experiments. *Significantly different at $p<0.05$ vs. control.

glutathione in cells, and it has been suggested that PEITC might inhibit $\mathrm{ABCC} 2$-mediated transport of other substances (25). Thus, the combined effect of PEITC with cisplatin appears to be based on suppression of $\mathrm{ABCC} 2$ expression and functional inhibition of $\mathrm{ABCC} 2$.

Although PXR is mainly a xenobiotic receptor that regulates the expression of drug-metabolizing enzymes and transporters to eliminate xenobiotics, it may also have a potential role in other cellular functions including apoptosis. PXR has been shown to be involved in the activation of the anti-apoptotic genes BCL2 apoptosis regulator (BCL2) and BCL2-like 1 (BCL2L1, previously BCL-XL) and to protect hepatocytes from staurosporine-induced apoptosis (26). Lin et al. demonstrated that in human osteosarcoma cells, ketoconazole induced cell death and apoptosis via c-Jun $\mathrm{N}$-terminal kinase (JNK) phosphorylation in a $\mathrm{Ca}^{2+}$-independent manner (27). Ketoconazole may have also a suppressive effect on the PXRregulated BCL2 family and proliferation signaling pathways, leading to a state in which cells are likely to undergo apoptosis. This action might lead to enhanced antitumor effect of cisplatin by ketoconazole.

In this study, we focused on PXR antagonists as agents capable of enhancing the antitumor activity of platinum compounds and compared the functional properties of ketoconazole and PEITC. Ketoconazole and PEITC, together with platinum compounds, resulted in increased caspase- 3 activity and intracellular platinum level, but no association was detected between caspase-3 activity and intracellular platinum level. Only in the presence of PEITC, which resulted in a marked increase in intracellular platinum level, was suppression of $\mathrm{ABCC} 2$ protein expression in cell membranes observed. On the other hand, ketoconazole resulted in a marked increase in caspase- 3 activity without major changes in the intracellular platinum level, indicating that the action of ketoconazole may be involved in the apoptotic control mechanism. These results suggested that ketoconazole and PEITC enhanced the antitumor activity of platinum compounds by suppressing different pathways regulated by PXR. Although PXR antagonists had greater effects when combined with platinum compounds, further studies will be required to determine how to use PXR antagonists that have multiple mechanisms of action, depending on the platinum compound to be used or the tumor to be targeted.

\section{Conflicts of Interest}

The Authors report no conflicts of interest.

\section{Authors' Contributions}

MY and SK conceived and designed the experiments; MY performed the experimental work; all Authors participated in data analysis; MY wrote the article; SK reviewed and revised the article. All Authors read and approved the final article.

\section{Acknowledgements}

The Authors would like to thank Editage (www.editage.jp) for English language editing.

\section{References}

1 Mackowiak B, Hodge J, Stern S and Wang H: The roles of xenobiotic receptors: Beyond chemical disposition. Drug Metab Dispos 46(9): 1361-1371, 2018. PMID: 29759961. DOI: 10.1124/dmd.118.081042

2 Lolodi O, Wang YM, Wright WC and Chen T: Differential regulation of CYP3A4 and CYP3A5 and its implication in drug discovery. Curr Drug Metab 18(12): 1095-1105, 2017. PMID: 28558634. DOI: $10.2174 / 1389200218666170531112038$

3 Hedrich WD, Hassan HE and Wang H: Insights into CYP2B6mediated drug-drug interactions. Acta Pharm Sin B 6(5): 413425, 2016. PMID: 27709010. DOI: 10.1016/j.apsb.2016.07.016 
$4 \mathrm{Hu}$ DG, Meech R, McKinnon RA and Mackenzie PI Transcriptional regulation of human UDP-glucuronosyltransferase genes. Drug Metab Rev 46(4): 421-458, 2014. PMID: 25336387. DOI: 10.3109/03602532.2014.973037

5 Tocchetti GN, Rigalli JP, Arana MR, Villanueva SSM and Mottino AD: Modulation of expression and activity of intestinal multidrug resistance-associated protein 2 by xenobiotics. Toxicol Appl Pharmacol 303: 45-57, 2016. PMID: 27155371. DOI: 10.1016/j.taap.2016.05.002

6 Pavek P: Pregnane X receptor (PXR)-mediated gene repression and cross-talk of PXR with other nuclear receptors via coactivator interactions. Front Pharmacol 7: 456, 2016. PMID: 27932985. DOI: $10.3389 /$ fphar.2016.00456

7 Ma X, Idle JR and Gonzalez FJ: The pregnane X receptor: From bench to bedside. Expert Opin Drug Metab Toxicol 4(7): 895908, 2008. PMID: 18624678. DOI: 10.1517/17425255.4.7.895

8 Wang T, Ma X, Krausz KW, Idle JR and Gonzalez FJ: Role of pregnane $\mathrm{X}$ receptor in control of all-trans retinoic acid (ATRA) metabolism and its potential contribution to ATRA resistance. $\mathrm{J}$ Pharmacol Exp Ther 324(2): 674-684, 2008. PMID: 17962516. DOI: $10.1124 /$ jpet.107.131045

9 Mensah-Osman EJ, Thomas DG, Tabb MM, Larios JM, Hughes DP, Giordano TJ, Lizyness ML, Rae JM, Blumberg B, Hollenberg PF and Baker LH: Expression levels and activation of a PXR variant are directly related to drug resistance in osteosarcoma cell lines. Cancer 109(5): 957-965, 2007. PMID: 17279585. DOI: $10.1002 / \mathrm{cncr} .22479$

10 Masuyama H, Nakatsukasa H, Takamoto N and Hiramatsu Y: Down-regulation of pregnane $\mathrm{X}$ receptor contributes to cell growth inhibition and apoptosis by anticancer agents in endometrial cancer cells. Mol Pharmacol 72(4): 1045-1053, 2007. PMID: 17636047. DOI: 10.1124/mol.107.037937

11 Amable L: Cisplatin resistance and opportunities for precision medicine. Pharmacol Res 106: 27-36, 2016. PMID: 26804248. DOI: $10.1016 /$ j.phrs.2016.01.001

12 Ghosh S: Cisplatin: The first metal-based anticancer drug. Bioorg Chem 88: 102925, 2019. PMID: 31003078. DOI: 10.1016/j.bioorg.2019.102925

13 Wen X, Buckley B, McCandlish E, Goedken MJ, Syed S, Pelis $\mathrm{R}$, Manautou JE and Aleksunes LM: Transgenic expression of the human MRP2 transporter reduces cisplatin accumulation and nephrotoxicity in Mrp2-null mice. Am J Pathol 184(5): 12991308, 2014. PMID: 24641901. DOI: 10.1016/j.ajpath.2014. 01.025

14 Han T, Zhu X, Wang J, Zhao H, Ma Q, Zhao J, Qiu X and Fan Q: Establishment and characterization of a cisplatin-resistant human osteosarcoma cell line. Oncol Rep 32(3): 1133-1139, 2014. PMID: 25017716. DOI: 10.3892/or.2014.3314

15 Kwatra D, Vadlapudi AD, Vadlapatla RK, Khurana V, Pal D and Mitra AK: Binary and ternary combinations of anti-HIV protease inhibitors: effect on gene expression and functional activity of CYP3A4 and efflux transporters. Drug Metabol Drug Interact 29(2): 101-110, 2014. PMID: 24399676. DOI: 10.1515/dmdi2013-0056.

16 Rigalli JP, Ciriaci N, Arias A, Ceballos MP, Villanueva SS, Luquita MG, Mottino AD, Ghanem CI, Catania VA and Ruiz ML: Regulation of multidrug resistance proteins by genistein in a hepatocarcinoma cell line: impact on sorafenib cytotoxicity PLoS One 10(3): e0119502, 2015. PMID: 25781341. DOI: 10.1371 /journal.pone. 0119502
17 Kwatra D, Venugopal A, Standing D, Ponnurangam S, Dhar A, Mitra A and Anant S: Bitter melon extracts enhance the activity of chemotherapeutic agents through the modulation of multiple drug resistance. J Pharm Sci 102(12): 4444-4454, 2013. PMID: 24129966. DOI: 10.1002/jps.23753

18 Yasuda M, Kishimoto S, Amano $M$ and Fukushima S: The involvement of pregnane $\mathrm{X}$ receptor-regulated pathways in the antitumor activity of cisplatin. Anticancer Res 39(7): 3601-3608, 2019. DOI: 10.21873/anticanres.13507

19 Maertens JA: History of the development of azole derivatives. Clin Microbiol Infect 10(Suppl 1): 1-10, 2004. PMID: 14748798.

20 Liebertz C and Fox P: Ketoconazole as a secondary hormonal intervention in advanced prostate cancer. Clin J Oncol Nurs 10(3): 361-366, 2006. PMID: 16789581. DOI: 10.1188/06. CJON.361-366

21 Huang H, Wang H, Sinz M, Zoeckler M, Staudinger J, Redinbo MR, Teotico DG, Locker J, Kalpana GV and Mani S: Inhibition of drug metabolism by blocking the activation of nuclear receptors by ketoconazole. Oncogene 26(2): 258-268, 2007. PMID: 16819505. DOI: 10.1038/sj.onc.1209788

22 Svecova L, Vrzal R, Burysek L, Anzenbacherova E, Cerveny L, Grim J, Trejtnar F, Kunes J, Pour M, Staud F, Anzenbacher P, Dvorak Z and Pavek P: Azole antimycotics differentially affect rifampicin-induced pregnane $\mathrm{X}$ receptor-mediated CYP3A4 gene expression. Drug Metab Dispos 36(2): 339-348, 2008. PMID: 17998298. DOI: $10.1124 / \mathrm{dmd} .107 .018341$

23 Zhou C, Poulton EJ, Grün F, Bammler TK, Blumberg B, Thummel KE and Eaton DL: The dietary isothiocyanate sulforaphane is an antagonist of the human steroid and xenobiotic nuclear receptor. Mol Pharmacol 71(1): 220-229, 2007. PMID: 17028159. DOI: $10.1124 / \mathrm{mol} .106 .029264$

24 Tang T, Song X, Liu YF and Wang WY: PEITC reverse multidrug resistance of human gastric cancer SGC7901/DDP cell line. Cell Biol Int 38(4): 502-510, 2014. PMID: 23956061. DOI: 10.1002/cbin.10169

$25 \mathrm{Ji}$ Y and Morris ME: Transport of dietary phenethyl isothiocyanate is mediated by multidrug resistance protein 2 but not $\mathrm{P}$ glycoprotein. Biochem Pharmacol 70(4): 640-647, 2005. PMID: 16002050. DOI: $10.1016 /$ j.bcp.2005.05.025

26 Zucchini N, de Sousa G, Bailly-Maitre B, Gugenheim J, Bars R, Lemaire $\mathrm{G}$ and Rahmani R: Regulation of BCL-2 and BCL-xL anti-apoptotic protein expression by nuclear receptor PXR in primary cultures of human and rat hepatocytes. Biochim Biophys Acta 1745(1): 48-58, 2005. PMID: 16085054. DOI: 10.1016/j.bbamcr.2005.02.005

27 Lin KL, Huang CC, Cheng JS, Tsai JY, Lu YC, Chang HT and Jan CR: Ketoconazole-induced JNK phosphorylation and subsequent cell death via apoptosis in human osteosarcoma cells. Toxicol In Vitro 23(7): 1268-1276, 2009. PMID: 19631732. DOI: $10.1016 /$ j.tiv.2009.07.025
Received June 28, 2019

Revised July 13, 2019

Accepted July 15, 2019 